\title{
Circulating tumor cells criteria (CyCAR) versus standard RECIST criteria for treatment response assessment in metastatic colorectal cancer patients
}

Mayte Delgado-Ureña ${ }^{1 \dagger}$, Francisco G. Ortega ${ }^{2 \dagger}$, Diego de Miguel-Pérez ${ }^{2,3 \dagger}$, Alba Rodriguez-Martínez ${ }^{1,2,3,4,5}$, Jose L. García-Puche1,2, Hugh Ilyine4, Jose A. Lorente2,3, Jose Exposito-Hernandez ${ }^{1}$, M. Carmen Garrido-Navas², Miguel Delgado-Ramirez ${ }^{5}$ and M. José Serrano ${ }^{1,2^{*}}$

\begin{abstract}
Background: The use of circulating tumor cells (CTCS) as indicators of treatment response in metastatic colorectal cancer (mCRC) needs to be clarified. The objective of this study is to compare the Response Evaluation Criteria in Solid Tumors (RECIST) with the Cytologic Criteria Assessing Response (CyCAR), based on the presence and phenotypic characterization of CTCS, as indicators of FOLFOX-bevacizumab treatment response.

Methods: 77 mCRC blood samples from FOLFOX-bevacizumab treated patients were analyzed to isolate CTCs before and after (12 and 24 weeks) treatment, using an immunomagnetic separation method. VEGFR expression was identified by double immunostaining.

Results: We observed a decrease of CTCS (42.8 vs. 18.2\%) and VEGFR positivity (69.7\% vs. $41.7 \%$ ) after treatment. According to RECIST, $6.45 \%$ of the patients did not show any clinical benefit, whereas $93.55 \%$ patients showed a favorable response at 12 weeks. According to CyCAR, 29\% had a non-favorable response and $71 \%$ patients did not. No significant differences were found between the response assessment by RECIST and CyCAR at 12 or 24 weeks. However, in the multivariate analysis, RECIST at 12 weeks and CyCAR at 24 weeks were independent prognostic factors for OS (HR: $0.1,95 \% \mathrm{Cl} 0.02-0.58$ and HR: $0.35,95 \% \mathrm{Cl} 0.12-0.99$ respectively).
\end{abstract}

Conclusions: CyCAR results were comparable to RECIST in evaluating the response in $\mathrm{mCRC}$ and can be used as an alternative when the limitation of RECIST requires additional response analysis techniques.

Keywords: Metastatic colorectal cancer, Bevacizumab, Circulating tumor cells, RECIST, CyCAR, Prognosis

\section{Background}

In colorectal cancer (CRC), metastasis is the main cause of death [1]. Distant metastasis is identified in

*Correspondence: mjose.serrano@genyo.es

${ }^{\dagger}$ Mayte Delgado-Ureña, Francisco G. Ortega and Diego de Miguel-Pérez contributed equally to this work

${ }^{2}$ Liquid Biopsy and Metastasis Research Group, GENYO, Centre

for Genomics and Oncological Research, Pfizer/University of Granada/ Andalusian Regional Government PTS, Granada, Avenida de la llustración,

114, 18016 Granada, Spain

Full list of author information is available at the end of the article approximately $25 \%$ of patients at initial diagnosis, and half of CRC patients will develop it [2]. During this process, circulating tumor cells (CTCs), detach from primary sites, enter the bloodstream and extravasate in distant organs. CTCs are now being studied in order to have a deeper understanding of the metastatic processes [3]. The phenotypic and genetic characterization of CTCs is especially important; as different subpopulations of CTCs can be detected in the blood of these patients [4]. These CTCs subclones can depict in real time the heterogeneity of a tumor, displaying its different abilities 
to elude therapies, and therefore, determining tumor response to treatment [5].

Metastatic colorectal cancer patients (mCRC) are currently subjected to a treatment regime combining chemotherapy with biological therapies. Bevacizumab, a monoclonal antibody inhibits the tyrosine kinase vascular endothelial growth factor A (VEGF-A) and blocks its transduction signal, through both VEGFR-1 and VEGFR2. VEGF-A is a potent pro-angiogenic growth factor that stimulates proliferation, migration, and survival of endothelial cells. As it is one of the more important proteins expressed by tumor cells, VEGF is an important target of anticancer therapies. Cancer cells and tissues with high metabolic rates are characterized by hypoxia, which induces the transcription of VEGF protein [6]. Circulating VEGF binds with high affinity to VEGF receptors (VEGFR-1 and VEGFR-2) and its co-receptors neuropilin (NRP-1 and NRP-2), which are expressed on the surface of endothelial cells and play a critical role in the development of angiogenesis, by stimulating recruitment and proliferation of endothelial cells [7]. Bevacizumab is an IgG1 recombinant humanized monoclonal antibody that acts by selectively binding to circulating VEGF-A, creating a large molecule that renders it unable to bind to its cell surface receptors, reducing microvascular growth of tumor blood vessels and limiting blood supply of nutrients and oxygen to tumor tissues. In combination with intravenous 5-fluorouracil-based chemotherapy, it is indicated for first- or second-line treatment of patients with metastatic colorectal cancer. In combination with fluoropyrimidine-irinotecan- or fluoropyrimidine-oxaliplatin-based chemotherapy, it is indicated for second-line treatment of patients with metastatic colorectal cancer who have progressed on a first-line Bevacizumab containing regimen $[8,9]$. First line bevacizumab has been demonstrated to improve overall survival (OS), progression-free survival (PFS) and treatment response rate in mCRC [10]. Despite these improvements, most mCRC patients will die due to disease progression [11]. With this in mind, extensive biomarker programs have now been built into numerous clinical studies with bevacizumab. However, predictive markers for bevacizumab treatment have yet to be validated [12].

In this clinical experimental work, we aimed to establish the predictive role of CTCs, and their expression of a treatment-associated marker (VEGFR), as response biomarkers to bevacizumab in mCRC patients, as well as their relationship with disease progression and death risk. Furthermore, we then compared the Response Evaluation Criteria in Solid Tumors (RECIST) version 1.1 [13] with our proposed Cytologic Criteria Assessing Response (CyCAR), based on CTC status, to determine their respective utility as predictive and prognostic assessments. Finally, we compared treatment responses of mCRC patients under FOLFOX-bevacizumab-containing chemotherapy by both criteria.

\section{Methods Study design}

We conducted a prospective longitudinal cohort study of 77 patients with mCRC who underwent first-line treatment with FOLFOX6 m (Oxaliplatin $85 \mathrm{mg} / \mathrm{m}^{2}$, Leucovorin $400 \mathrm{mg} / \mathrm{m}^{2}, 5$-FU $400 \mathrm{mg} / \mathrm{m}^{2}$ bolus and 5 -FU $2400 \mathrm{mg} / \mathrm{m}^{2}$ over $46 \mathrm{~h}$ ) and bevacizumab $(5 \mathrm{mg} / \mathrm{kg})$ every 2 weeks until disease progression, at the Department of Oncology, San Cecilio University Hospital in Granada (Spain), between April 2011 and November 2015. Control blood samples were drawn from 16 healthy volunteers with no history of malignant disease.

The study was conducted in accordance with the Declaration of Helsinki and approved by the ethical Committee of the Hospital. Written informed consent was obtained from every cancer patient and healthy volunteer.

None of the patients had received any other type of biological treatment before inclusion in the study. Computed tomography of the chest, abdomen and pelvis was performed at baseline, at 12 weeks, at 24 weeks and finally each 12 weeks until death. Image interpretation was performed using RECIST, to classify each disease as complete response, partial response, stable disease, or progressive disease. Patients who died before a followup imaging study were classified as having progressive disease. According to patient response to the therapy, they were divided into two groups: those with favorable response, including patients who have achieved complete response, partial response or stable disease, and those with non-favorable response, including patients without clinical benefit (progressive disease or death).

Median follow-up time for all patients was 23.3 months (range 2-105 months). Clinical outcomes were evaluated in terms of PFS and OS. PFS was defined as the elapsed time from the start of the treatment to progression or death. OS was defined as the elapsed time from the start of the treatment to death.

Data was collected for the following variables: age, gender, primary tumor location, metastasis surgery, primary tumor surgery, synchronous metastasis, K-RAS status, Basal CEA, Basal Ca 19.9, progression, survival, RECIST and CyCAR responses (Table 1 ).

CYCAR results were based on the status of CTCs during the treatment (Fig. 1). Thus, a patient was classified as a responder (favorable response) if he was negative at baseline and continued negative for CTCs at 12 weeks. However, a patient was classified as a non-responder (non-favorable response) if he showed CTCs at 12 weeks. In the same way, the patient was classified as responder 
Table 1 Clinicopathological characteristics of the patients according to the CyCAR criteria at baseline status (CTC1) and VEGFR status

\begin{tabular}{|c|c|c|c|c|c|c|}
\hline & \multicolumn{3}{|l|}{ CTC 1} & \multicolumn{3}{|c|}{ CTC 1 VEGFR } \\
\hline & N (\%) - & $\mathrm{N}(\%)+$ & $p$ & N (\%) - & $\mathrm{N}(\%)+$ & $p$ \\
\hline \multicolumn{7}{|l|}{ Age (years) } \\
\hline$<55$ & $15(62.5 \%)$ & $9(37.5 \%)$ & 0.622 & $3(33.3 \%)$ & $6(66.7 \%)$ & 1.00 \\
\hline$\geq 55$ & $29(54.7 \%)$ & $24(45.3 \%)$ & & $7(29.2 \%)$ & $17(70.8 \%)$ & \\
\hline \multicolumn{7}{|l|}{ Gender } \\
\hline Male & $30(58.2 \%)$ & $21(41.2 \%)$ & 0.808 & $9(40.9 \%)$ & $13(59.1 \%)$ & 0.144 \\
\hline Female & $14(53.9 \%)$ & $12(46.1 \%)$ & & $2(16.7 \%)$ & $10(83.3 \%)$ & \\
\hline \multicolumn{7}{|c|}{ Primary tumor location } \\
\hline Colon & $25(54.4 \%)$ & $21(45.6 \%)$ & 0.641 & $5(23.8 \%)$ & $16(76.2 \%)$ & 0.164 \\
\hline Rectum & $19(61.3 \%)$ & $12(38.7 \%)$ & & $6(46.2 \%)$ & $7(53.8 \%)$ & \\
\hline \multicolumn{7}{|c|}{ Metastasis surgery } \\
\hline No & $32(57.1 \%)$ & $24(42.9 \%)$ & 1.00 & $8(32 \%)$ & $17(68 \%)$ & 0.625 \\
\hline Yes & $12(57.1 \%)$ & $9(42.9 \%)$ & & $3(33.3 \%)$ & $6(66.7 \%)$ & \\
\hline \multicolumn{7}{|c|}{ Primary tumor surgery } \\
\hline No & $21(75 \%)$ & $7(25 \%)$ & $0.019^{*}$ & $4(57.1 \%)$ & $3(42.9 \%)$ & 0.132 \\
\hline Yes & $23(46.9 \%)$ & $26(53.1 \%)$ & & $7(25.9 \%)$ & $20(74.1 \%)$ & \\
\hline \multicolumn{7}{|c|}{ Synchronous metastasis } \\
\hline No & $3(25 \%)$ & $9(75 \%)$ & $0.024^{*}$ & $2(22.2 \%)$ & $7(77.8 \%)$ & 0.686 \\
\hline Yes & $41(63.1 \%)$ & $24(36.9 \%)$ & & $8(33.3 \%)$ & $16(66.7 \%)$ & \\
\hline \multicolumn{7}{|l|}{ K-RAS } \\
\hline Mutated & $25(69.4 \%)$ & $11(30.6 \%)$ & 0.061 & $4(36.4 \%)$ & $7(63.6 \%)$ & 0.546 \\
\hline Wild-type & $18(46.2 \%)$ & $21(53.8 \%)$ & & $7(31.8 \%)$ & $22(68.2 \%)$ & \\
\hline \multicolumn{7}{|l|}{ Basal CEA } \\
\hline Standard & $6(40 \%)$ & $9(60 \%)$ & 0.153 & $2(20 \%)$ & $8(80 \%)$ & 0.339 \\
\hline High & $37(61.7 \%)$ & $23(38.3 \%)$ & & $8(34.8 \%)$ & 15 (65.2\%) & \\
\hline \multicolumn{7}{|l|}{ Basal Ca 19.9} \\
\hline Standard & $19(46.3 \%)$ & $22(53.7 \%)$ & $0.034^{*}$ & $7(31.8 \%)$ & $15(68.2 \%)$ & 0.627 \\
\hline High & $23(71.9 \%)$ & $9(28.1 \%)$ & & $3(30 \%)$ & $7(70 \%)$ & \\
\hline \multicolumn{7}{|l|}{ Progression } \\
\hline Yes & $28(53.8 \%)$ & $24(46.2 \%)$ & 0.485 & 7 (29.2\%) & $17(70.8 \%)$ & 0.634 \\
\hline No & $9(64.3 \%)$ & $5(35.7 \%)$ & & $2(40 \%)$ & $3(60 \%)$ & \\
\hline \multicolumn{7}{|l|}{ Survival } \\
\hline Yes & $18(60 \%)$ & $12(40 \%)$ & 1.00 & $3(25 \%)$ & $9(75 \%)$ & 0.683 \\
\hline No & $22(61.1 \%)$ & $14(38.9)$ & & $5(35.7 \%)$ & $9(64.3 \%)$ & \\
\hline \multicolumn{7}{|c|}{ RECIST 12 weeks } \\
\hline Favorable & 31 (53.4\%) & $27(46.6 \%)$ & 0.402 & $7(25.9 \%)$ & $20(74.1 \%)$ & 0.557 \\
\hline Adverse & $3(75 \%)$ & $1(25 \%)$ & & $0(0 \%)$ & $1(100 \%)$ & \\
\hline \multicolumn{7}{|c|}{ RECIST 24 weeks } \\
\hline Favorable & $19(52.8 \%)$ & $17(47.2 \%)$ & 0.376 & $4(23.5 \%)$ & $13(76.5 \%)$ & 0.468 \\
\hline Adverse & $5(38.5 \%)$ & $8(61.5 \%)$ & & $3(37.5 \%)$ & $5(62.5 \%)$ & \\
\hline \multicolumn{7}{|c|}{ CyCAR 12 weeks } \\
\hline Favorable & $29(65.9 \%)$ & 15 (34.1\%) & 0.087 & $4(26.7 \%)$ & $11(73.3 \%)$ & 1.00 \\
\hline Adverse & 7 (38.9\%) & 11 (61.1\%) & & $3(27.3 \%)$ & $8(72.7 \%)$ & \\
\hline \multicolumn{7}{|c|}{ CyCAR 24 weeks } \\
\hline Favorable & $30(55.6 \%)$ & $24(44.4 \%)$ & 0.537 & $7(29.2 \%)$ & $17(70.8 \%)$ & 0.574 \\
\hline Adverse & 8 (66.7\%) & 4 (33.3\%) & & $2(50 \%)$ & $2(50 \%)$ & \\
\hline
\end{tabular}

*: Statistically significant

CyCAR: Cytologic Criteria Assessing Response; CTC: circulating tumor cell; VEGFR: vascular endothelial growth factor receptor; RECIST: Response Evaluation Criteria in Solid Tumors; $p$ : $p$ value 


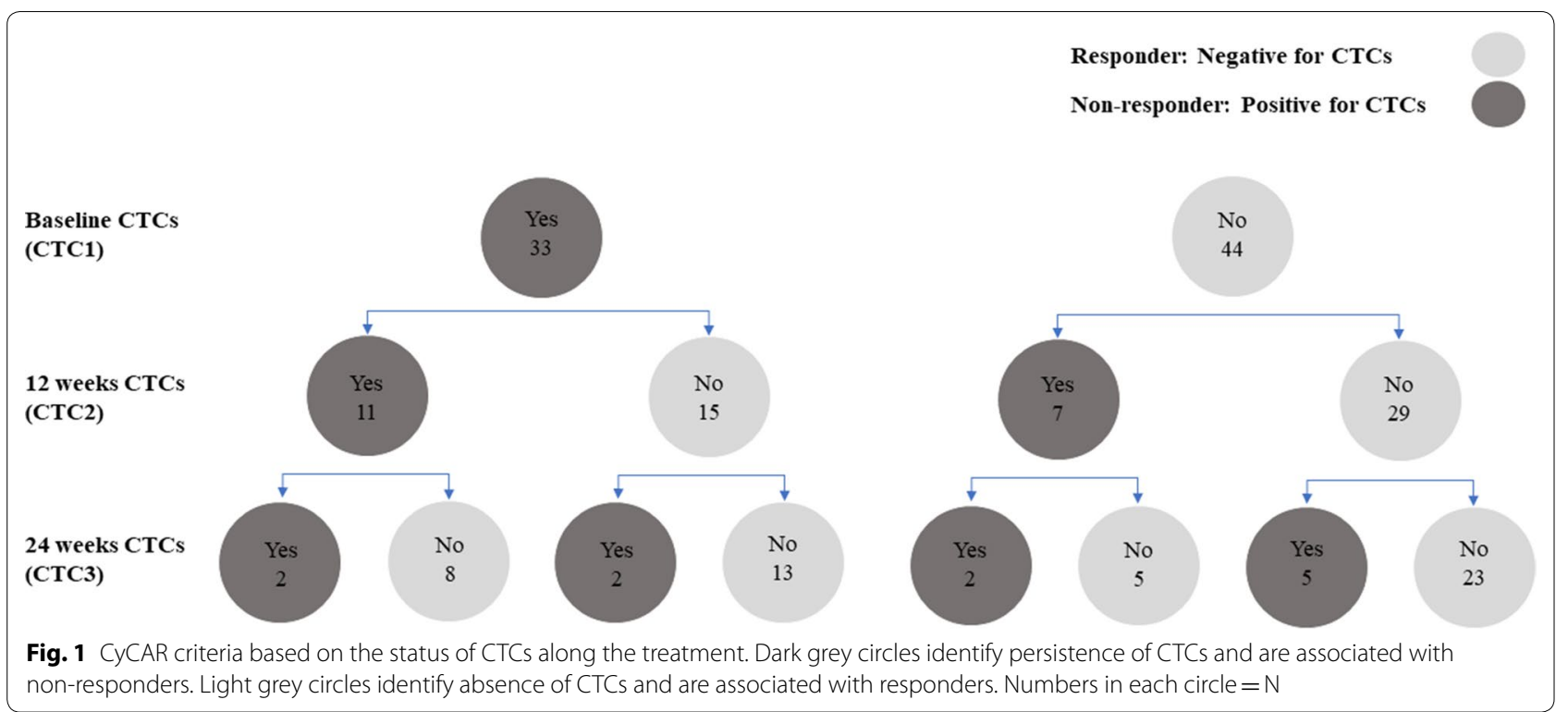

if he was negative for CTCs at 24 weeks, but he was classified as non-responder if he showed CTCs at 24 weeks.

\section{Isolation and enumeration of CTCs}

Peripheral blood was collected for CTCs evaluation before the initiation of therapy (baseline) (CTC1) and subsequently at 12 (CTC2), and 24 (CTC3) weeks after initiating the treatment.

For CTCs enrichment and detection, $10 \mathrm{ml}$ of peripheral blood was collected from each $\mathrm{mCRC}$ patient and processed according to the protocols based on immunomagnetic selection and established by our group [14].

\section{Enumeration and characterisation of CTCs by CK and VEGFR expression}

Samples containing $\mathrm{CTCs}^{\mathrm{CK}+}$ were characterized for VEGFR expression by double immunofluorescence (IF) following our standard protocols [14]. We also analyzed 17 healthy blood donors and a colon cancer cell line (HT29) to test the performance of the assays.

\section{Fluorescence microscopy}

Cytospins were previously analyzed for the presence of CTCs under direct light microscope to identify red stained pan-CK cells. Then, samples were observed using a computerized fluorescence microscope Zeiss AXIO Imager. A1 to detect cells with intense VEGFR staining. VEGFR positive cells $\left(\mathrm{CTCs}^{\mathrm{CK}+\mathrm{VEGFR}+}\right)$ showed an intense blue fluorescence signal on the surface (Fig. 2).

\section{Statistical analysis}

CTCs were assessed as a continuous and a binary variable (presence/absence). The cut-off limit for CTC status as positive was $\mathrm{CTCs} \geq 1$. The relationships between CTCs and other variables were ascertained using Fisher's exact test. The Wilcoxon signed-rank test was used to compare CTCs measured at two different times and the Cochran's Q test to compare presence of CTCs at three different times. The influence of clinicopathological variables on PFS and OS was measured by univariate and multivariate Cox Proportional-Hazards Regression. We applied the criterion of more than a $10 \%$ change in the CTC coefficient estimate [15] for the selection of variables to be included in the multivariate model.

\section{Results}

The study was performed in 77 patients with metastatic cancer of colon and rectum $(59.7 \%$ and $40.3 \%$ respectively). The median age was 61 years and $51 \%$ of patients were men. Only 12 patients (15.6\%) presented synchronous metastases while $65(84.4 \%)$ presented metachronous metastases (metastases developed at least 12 months after the primary tumor) (Table 1). In this way, 59 (76.6\%) patients developed liver metastasis, $8(10.4 \%)$ lung metastasis and the remaining $10(13 \%)$ developed metastasis in other organs as bone, lymph nodes or adrenal glands. Nevertheless, no relation was found between levels or status of CTCs and a predisposition to metastasis between organs $(\mathrm{p}>0.05)$. 


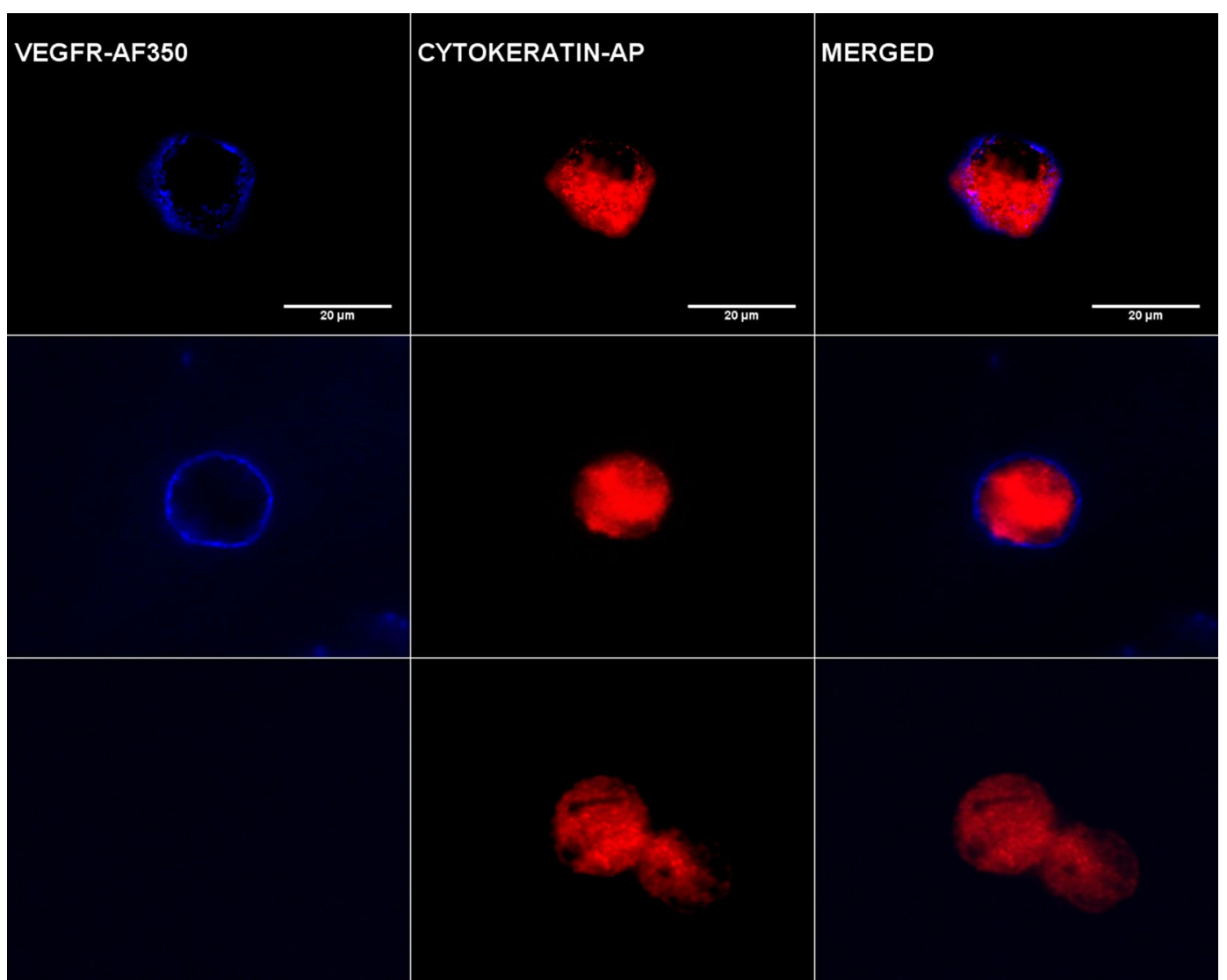

Fig. 2 Image gallery after isolation, cytomorphological analysis and detection of cytokeratin-positive tumor cells (CK+, red staining) and vascular endothelial growth factor expression (VEGFR, blue staining). Top row shows HT29 cell tumor line used as a positive control for VEGFR expression. Middle (patient 1) and bottom (patient 2) rows show an example of heterogeneous expression of VEGFR in two different patients: patient 1 shows positive VEGFR expression in a CTC and patient 2 shows negative VEGFR expression in another CTC. VEGFR-specific immunofluorescence (IF) CTCs were determined with Alexa Fluor ${ }^{\circledR} 350$

\section{Dynamic fluctuation of CTCs during follow-up} and correlation with clinicopathological characteristics

CTCs were detected in 33 of $77(42.8 \%)$ patients at CTC1, in 18 of $62(29 \%)$ patients at CTC2 and finally, in 12 of $66(18.2 \%)$ patients at CTC3 (blood samples of some patients were lost due principally to the death of the patients, except for two of them, which could not be analyzed due to sample analysis problems). According to the data, we observed a significant decrease in number of patients with CTCs along the follow-up (CTC1CTC3) $(\mathrm{p}=0.015)$ and between extraction points CTC1 vs. CTC2 (0.019) and CTC1 vs. CTC3 (0.003). This way, the mean number of CTCs varied from an initial 1.5 cells per $10 \mathrm{ml}$ of peripheral blood [standard deviation (SD): 1.5; range 0-8] at CTC1, 1.7 cells (SD: 8.1; range 0-64) at CTC2, to a reduced number of 0.5 (SD: 1.5 ; range $0-9$ ) at CTC3 (Table 2).

Correlation between CTC1 presence, CTC1 ${ }^{\mathrm{VEGFR}}$ status and clinic-pathological characteristics is summarized in Table 1 . The primary tumor resection showed a significant correlation with CTC1 presence $(p=0.019)$. 26 of $49(53.1 \%)$ patients who underwent primary tumor resection were CTC1+, while only 7 of $28(25 \%)$ patients without primary tumor resection were CTC1. Despite finding higher percentage of $\mathrm{CTC} 1+$ patients with wildtype K-RAS tumors than patients with mutated K-RAS tumors ( $54 \%$ vs. $31 \%$ respectively), there was no significant correlation between them $(p=0.061)$. Lower levels of basal Ca 19.9 significantly correlated with CTC1 presence $(p=0.034)$. Therefore, 22 of $41(53.7 \%)$ patients with standard levels of Ca 19.9 showed CTC1 presence but only 9 of $32(28.1 \%)$ did it in the higher levels group (Table 1).

\section{Dynamic fluctuation of CTCs according to VEGFR status} and correlation with clinic-pathological characteristics At baseline status, CTC1 $1^{\mathrm{CK}+\mathrm{VEGFR}+}$ were detected in 23 of 33 (69.7\%) patients. At the second extraction, we found $\mathrm{CTC} 22^{\mathrm{CK}+\mathrm{VEGFR}+}$ in 7 of 18 (38.9\%) patients, and finally, we found $\mathrm{CTC} 3^{\mathrm{CK}+\mathrm{VEGFR}+}$ in 5 of $12(41.7 \%)$ 
Table 2 Dynamic fluctuation of CTCs in patients according to VEGFR status during follow-up

\begin{tabular}{|c|c|c|c|c|c|c|}
\hline & CTC1 (baseline) & CTC2 (12 weeks) & CTC3 (24 weeks) & $p$ (CTC1 vs CTC2) & $p$ (CTC1 vs CTC3) & $p$ (CTC1-CTC3) \\
\hline Patients with CTCs $\left(\mathrm{CTCs}^{\mathrm{CK}+}\right) \mathrm{N}(\%)$ & $33(42.8 \%)$ & $18(29 \%)$ & $12(18.2 \%)$ & 0.088 & 0.005 & 0.015 \\
\hline $\begin{array}{l}\text { Mean number of } \mathrm{CTCS}^{\mathrm{CK}+} \text { (SD; } \\
\text { range) }\end{array}$ & $1.5(1.5 ; 0-8)$ & $1.7(8.1 ; 0-64)$ & $0.5(1.5 ; 0-9)$ & 0.019 & 0.003 & \\
\hline Patients with CTCs ${ }^{\mathrm{CK}+\mathrm{VEGFR+}}$ ) N (\%) & $23(69.7 \%)$ & $7(38.9 \%)$ & $5(41.7 \%)$ & 0.102 & 0.317 & 0.368 \\
\hline $\begin{array}{l}\text { Mean number of CTCs }{ }^{\text {CK+VEGFR+ }} \\
\text { (SD; range) }\end{array}$ & $1.4(1.5 ; 0-5)$ & $0.6(1.05 ; 0-3)$ & $0.5(0.8 ; 0-2)$ & 0.120 & 0.317 & \\
\hline
\end{tabular}

CTCs: circulating tumor cells; CK: cytokeratin; VEGFR: vascular endothelial growth factor receptor; SD: standard deviation; $p: p$ value

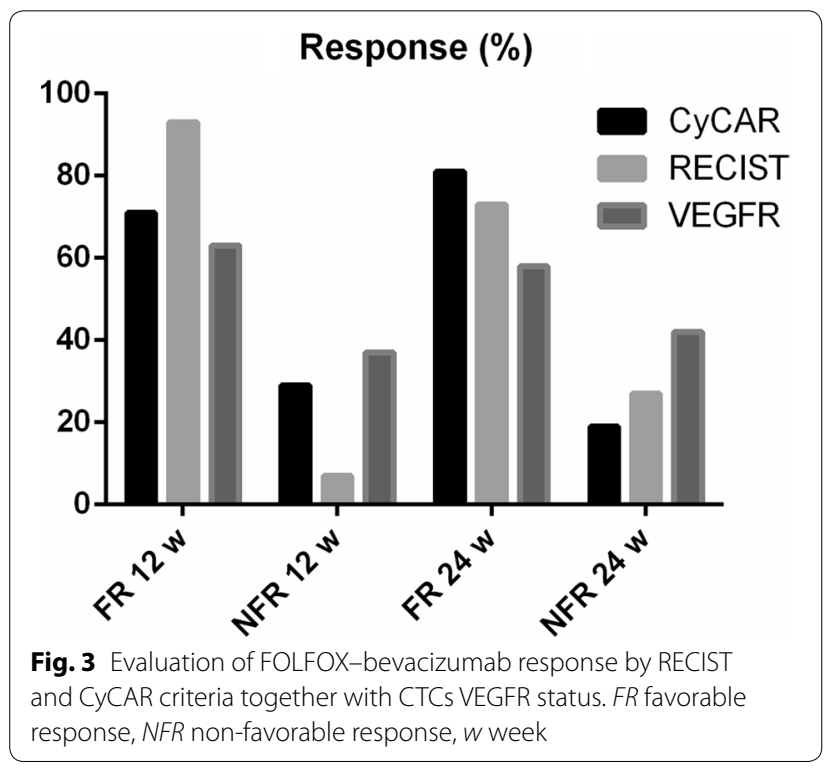

patients. The mean number of $\mathrm{CTC} 1^{\mathrm{CK}+\mathrm{VEGFR}+}$ was 1.4 per $10 \mathrm{ml}$ of peripheral blood (SD: 1.5 ; range $0-5$ ), $0.6 \mathrm{CTC} 2 \mathrm{CK}+\mathrm{VEGFR}+{ }^{\mathrm{SD}}: 1.05$; range $\left.0-3\right)$ and 0.5 CTC3 ${ }^{\mathrm{CK}+\mathrm{VEGFR}+}$ (SD: 0.8; range 0-2) (Table 2). During follow-up, although it was not statistically significant, we noted a decrease not only in the percentage of patients with $\mathrm{CTC}^{\mathrm{CK}+\mathrm{VEGFR+}}$, but also in the number of CTC $\mathrm{CK}^{\mathrm{V}+\mathrm{VEGFR}+}$ detected per patient (Table 2). However, no significant correlation was found between $\mathrm{CTC1} 1^{\mathrm{CK}+\mathrm{VEGFR}+}$ status and any clinic-pathological characteristics of the patients, including K-RAS status (Table 1).

We compared the previously described CyCAR results to RECIST, in order to evaluate the response at 12 and 24 weeks. We evaluated responses in 62 patients at 12 weeks (Fig. 3). According to RECIST, 4 of the $62(6.45 \%)$ patients showed non-favorable responses, while 58 of the $62(93.55 \%)$ patients showed a favorable response. The CyCAR (CTC2) responses showed similar results, with 18 of the 62 patients (29\%) developing non-favorable responses and 44 of the $62(71 \%)$ patients developing a favorable one. As a result, we found no significant differences between the assessment response by RECIST and CyCAR at 12 weeks $(p>0.05)$.

At 24 weeks, we evaluated responses in 65 patients. In this case, according to RECIST, 13 of the 65 (20\%) patients showed non-favorable responses, while 52 of them $(80 \%)$ showed a favorable response. On the other hand, regarding CyCAR evaluation (CTC3), 12 of the 65 patients (18.5\%) showed non-favorable responses while 53 of them (81.5\%) showed a favorable response. In the same way, we found no significant differences between both assessment criteria $(p>0.05)$.

\section{Correlation between RECIST and CyCAR based on CTCs VEGFR status to evaluate response to FOLFOX- bevacizumab}

CyCAR results based on VEGFR status in CTC2 and CTC3 was compared to RECIST evaluation at 12 and 24 weeks. At the first response evaluation time point (12 weeks), 18 patients were analyzed, while at the second response re-evaluation (24 weeks), only 12 of the 18 patients remained $\mathrm{CTC} 3+$ and were analyzed (Table 2).

According to RECIST at 12 weeks, 17 of the 18 (94.4\%) patients showed favorable responses and only 1 of these 18 patients (5.6\%) developed a non-favorable response. Using CyCAR, 7 of the $18 \mathrm{CTC} 2{ }^{\mathrm{CK}+}$ patients were VEGFR+, with 6 of the 7 (85.71\%) patients with CTC2 ${ }^{\mathrm{CK}+\mathrm{VEGFR}+}$ showing favorable responses, and just 1 of the $7(14.29 \%)$ patients showing a non-favorable response. The remaining $11 \mathrm{CTC} 2^{\mathrm{CK}+\mathrm{VEGFR}-}$ patients were identified as responders $(p>0.05)$ (Fig. 3).

On the other hand, according to RECIST, at 24 weeks 9 of the 12 (75\%) CTC3+ patients showed favorable responses with only 3 of them (25\%) developing a non-favorable response. According to CyCAR, 5 of the $12(42 \%)$ patients were CTC3 ${ }^{\mathrm{CK}+\mathrm{VEGFR}+}$ and all of them showed a favorable response. However, in the CTC3 ${ }^{\text {CK+VEGFR- }}$ group (7/12), 4 (57\%) were identified as responders and three as non-responders $(43 \%)$, showing no statistical differences between the groups $(p>0.05)$. 


\section{Prognostic significance of CTCs detection}

36 of the total 74 patients enrolled in this study died from disease progression. Median overall survival for these 36 patients was 18.3 months, compared to 37.5 months for the remaining 38 patients.

In the univariate analysis of the factors associated with OS (Table 3), we did not find any positive association between RECIST response at 12 weeks and risk of death (HR: $0.26,95 \%$ CI $0.06-1.14 ; p>0.05$ ). In the same way, there was no correlation between CyCAR at 12 weeks (CTC2) and risk of death (HR: 1.36; 95\% CI 0.62-3.01; $p>0.05)$.

At 24 weeks, we found no positive correlation between the risk of death and CyCAR and RECIST criteria (HR: 0.221 ; $95 \%$ CI $0.22-1.12 ; p>0.05$ and HR: $0.5 ; 95 \%$ CI $0.22-1.26 ; p>0.05$, respectively).

Then, we analyzed the OS in responder and nonresponder patients by RECIST and CyCAR. We observed that the median $\mathrm{OS}$ in responder vs. non-responder patients was 48.6 vs. 14.8 months according to RECIST and 55.4 vs. 22.4 months by CyCAR, evaluated at 24 weeks. In conjunction with these results, the presence of CTCs after treatment identified those patients with worse OS, which was in concordance with the results obtained by RECIST.

In the univariate analyses, metastasis surgery was significantly associated with a higher overall survival $(\mathrm{HR}=0.35 ; 95 \%$ CI $0.15-0.81 ; p=0.014)$. In the same way, we found that the presence of synchronous metastasis decreased the OS when compared to the presence of metachronous metastasis $(\mathrm{HR}=15.89$; $95 \%$ CI $2.04-$ $123.35 ; p=0.008$ ).

The multivariate analysis included the significant risk factors from the univariate analysis. Response assessment at 12 weeks by RECIST, response assessment at 24 weeks by CyCAR, and synchronous metastasis variables were independent prognostic factors associated with OS (Table 3). According to the PFS, RECIST response at 12 weeks was the only significantly associated variable in the multivariate analysis.

\section{Discussion}

RECIST has been adopted as the standard method for tumor assessment and helps in clinical decision making. Deciding and then monitoring the effectiveness of individual therapies in mCRC patients is currently very challenging, as a result of the high prevalence of lymph abdominal, peritoneal, serous and pleural metastasis, which are particularly difficult to evaluate by RECIST [16]. In addition, the thresholds (of response or progression) for predicting differences in survival in treated patients probably differ according to the type of treatment and the type of cancer [17]. For example, targeted molecules such as anti-VEGF or anti-EGFR often induce only small size changes, whereas patient survival is significantly prolonged. Therefore, specific criteria for certain diseases or treatments are necessary, as the current biomarkers and imaging evaluation options to monitor and register treatment clinical responses do not yet allow for optimal management of individual patients yet [18].

The hypothesis that CTCs are a fundamental prerequisite to metastasis was first proposed in the midnineteenth century (1869) by Thomas Ashworth, an Australian pathologist [19]. The characterization of CTCs, derived from a 'simple' blood test, have the potential to serve as 'real-time tumor biopsies' permitting accurate, up-to-date pictures of tumor activity, without the need for invasive tissue biopsies. Furthermore, CTCs can be analyzed on a serial basis, allowing real-time identification of emerging treatment 'resistance profiles', and consequently, being of significant assistance to the radiological assessment of tumor responses [20].

We performed this study in 77 patients with metastatic colorectal cancer treated in a homogeneous manner. The overall response rate to treatment was 24 months and median overall survival was 23.3 months, which did not differ from that expected [21]. In our study, we cover responses of metastatic colon cancer patients to bevacizumab, assessing and comparing CTCs and standard evaluation criteria, and we found comparable results to other published studies.

In our study, we compared CyCAR to RECIST in the same mCRC patient cohort, to determine the specific importance of CyCAR. In addition, we analyzed the association between OS and PFS with different clinic-pathological characteristics such as the presence of CTCs, at follow-up times.

The correlation between K-RAS tumor status and the presence of CTCs was analyzed, finding borderline significant association $(p=0.06)$ between higher percentages of CTC positive patients with K-RAS wild-type tumors. Similar results were reported in a recent study with 24 metastatic colon cancer patients by Das et al. [22]. In the same way, Buim et al. [23] observed that only 9 of their 23 CTCs positive patients had K-RAS mutations in their corresponding primary tumors. Our results, together with the aforementioned studies, suggest that the dissemination of CTCs is an independent process to K-RAS status in the primary tumor.

On the other hand, we detected a direct association between the presence of CTCs and primary tumor surgery. In fact, most patients undergoing primary tumor surgical treatment were positive for the presence of CTCs. These results concur with several studies, which demonstrated that primary tumor resection can stimulate cellular proliferation of residual colorectal tumors [24]. 
Table 3 Univariate and multivariate Cox regression analysis for overall survival (OS)

\begin{tabular}{|c|c|c|c|c|c|c|c|}
\hline \multirow[t]{2}{*}{ Characteristics } & \multicolumn{4}{|c|}{ Univariate analysis } & \multicolumn{3}{|c|}{ Multivariate analysis } \\
\hline & $\begin{array}{l}\text { Median OS } \\
\text { (months) }\end{array}$ & HR & $95 \% \mathrm{Cl}$ & $p$ & HR & $95 \% \mathrm{Cl}$ & $p$ \\
\hline All patients & 30.3 & & & & & & \\
\hline \multicolumn{8}{|l|}{ Age } \\
\hline$<55$ & 22.4 & 1.72 & $0.88-3.37$ & 0.113 & & & \\
\hline$\geq 55$ & 23.4 & & & & & & \\
\hline \multicolumn{8}{|l|}{ Gender } \\
\hline Male & 23.3 & 0.70 & $0.36-1.38$ & 0.307 & & & \\
\hline Female & 20 & & & & & & \\
\hline \multicolumn{8}{|c|}{ Primary tumor location } \\
\hline Colon & 22.4 & 0.78 & $0.39-1.58$ & 0.497 & & & \\
\hline Rectum & 22.85 & & & & & & \\
\hline \multicolumn{8}{|c|}{ Primary tumor surgery } \\
\hline Yes & 39.4 & 0.17 & $0.08-0.37$ & $<0.0001$ & 0.37 & $0.12-1.11$ & 0.075 \\
\hline No & 11.6 & & & & & & \\
\hline \multicolumn{8}{|c|}{ Response ( 12 weeks) (RECIST) } \\
\hline Favorable & 41.6 & 0.26 & $0.06-1.14$ & 0.074 & 0.1 & $0.02-0.58$ & $0.011^{*}$ \\
\hline Adverse & 16.8 & & & & & & \\
\hline \multicolumn{8}{|c|}{ Response (24 weeks) (RECIST) } \\
\hline Favorable & 48.6 & 0.51 & $0.22-1.20$ & 0.123 & & & \\
\hline Adverse & 14.8 & & & & & & \\
\hline \multicolumn{8}{|l|}{ K-RAS status } \\
\hline Mutated & 36.9 & 1.95 & $0.96-3.97$ & 0.064 & 1.58 & $0.68-3.68$ & 0.289 \\
\hline Wild-type & 55.4 & & & & & & \\
\hline \multicolumn{8}{|l|}{ Metastasis surgery } \\
\hline Operated & 68.4 & 0.35 & $0.15-0.81$ & 0.014 & 0.39 & $0.12-1.24$ & 0.111 \\
\hline Non-operated & 31.1 & & & & & & \\
\hline \multicolumn{8}{|c|}{ Synchronous metastasis } \\
\hline Yes & 32.1 & 15.89 & $2.04-123.35$ & 0.008 & 18.33 & $1.52-221.18$ & $0.022^{*}$ \\
\hline No & 68.4 & & & & & & \\
\hline \multicolumn{8}{|l|}{ CEA Basal } \\
\hline High & 39.4 & 1.96 & $0.84-4.57$ & 0.121 & & & \\
\hline Standard & 55.4 & & & & & & \\
\hline \multicolumn{8}{|l|}{ Basal Ca 19.9} \\
\hline High & 22.4 & 1.68 & $0.87-3.24$ & 0.123 & & & \\
\hline Standard & 55.4 & & & & & & \\
\hline \multicolumn{8}{|l|}{ СTC1 } \\
\hline Favorable & 21.6 & 0.32 & $0.72-2.79$ & 0.319 & & & \\
\hline Adverse & 23.5 & & & & & & \\
\hline \multicolumn{8}{|l|}{ CTC1 VEGFR } \\
\hline Yes & 66.5 & 0.53 & $0.25-1.15$ & 0.109 & & & \\
\hline No & 36.9 & & & & & & \\
\hline \multicolumn{8}{|l|}{ СTC2 } \\
\hline Favorable & 39 & 1.36 & $0.62-3.01$ & 0.442 & & & \\
\hline Adverse & 55.4 & & & & & & \\
\hline \multicolumn{8}{|l|}{ CTC2 VEGFR } \\
\hline Yes & 36.9 & 1.44 & $0.31-6.66$ & 0.640 & & & \\
\hline No & 41.6 & & & & & & \\
\hline
\end{tabular}


Table 3 (continued)

\begin{tabular}{|c|c|c|c|c|c|c|c|}
\hline \multirow[t]{2}{*}{ Characteristics } & \multicolumn{4}{|c|}{ Univariate analysis } & \multicolumn{3}{|c|}{ Multivariate analysis } \\
\hline & $\begin{array}{l}\text { Median OS } \\
\text { (months) }\end{array}$ & $H R$ & $95 \% \mathrm{Cl}$ & $p$ & HR & $95 \% \mathrm{Cl}$ & $p$ \\
\hline \multicolumn{8}{|l|}{ CTC3 } \\
\hline Favorable & 55.4 & 0.52 & $0.22-1.26$ & 0.149 & 0.35 & $0.12-0.99$ & $0.049^{*}$ \\
\hline Adverse & 22.4 & & & & & & \\
\hline \multicolumn{8}{|l|}{ CTC3 VEGFR } \\
\hline Yes & 22.4 & 1.04 & $0.23-4.81$ & 0.958 & & & \\
\hline No & 30.9 & & & & & & \\
\hline
\end{tabular}

*: Statistically significant

CTC: circulating tumor cell; OS: overall survival; HR: hazard risk; Cl: confidence interval; VEGFR: vascular endothelial growth factor receptor positive; RECIST: Response Evaluation Criteria in Solid Tumors; $p$ : $p$ value

However, other studies demonstrated that primary tumor resection improves OS in metastatic colon patients [25]. Interestingly, the absence of CTCs in mCRC has been also associated with a higher OS in several studies [26].

Additionally, besides analyzing the presence of CTCs, we also analyzed their heterogeneous VEGFR expression. VEGF is the target of bevacizumab, a humanized anti-VEGF monoclonal antibody (Bevacizumab; Avastin). This drug has been approved by the FDA in the first and second-line colorectal cancer setting, in combination with chemotherapy. Bevacizumab acts by selectively binding circulating VEGF, inhibiting the binding of VEGF to its cell surface receptor, and in this way preventing its activation. Despite this immobilization of VEGF, we found no association between VEGFR status in CTCs with the response to treatment, even though we describe a no statistically significant reduction in the cell subpopulations of CTC ${ }^{\mathrm{CK}+\mathrm{VEGFR}+}$ and a decrease in the number of CTC ${ }^{\mathrm{CK}+\mathrm{VEGFR}+}$ patients, before and after treatment. These results suggest that the treatment could be effective at removing VEGFR+ tumor cell populations, but also selecting VEGFR - populations. Interestingly, Simiantonaki et al. [27] demonstrated that negative VEGFR-1 expression was significantly associated with lymphogenous and haematogenous metastases. In the same way, Hanrahan et al. [28] found a significant increase of VEGFR-1 mRNAs in T3/T4 colorectal carcinomas compared to lymphogenously metastasising tumors, and Lebok et al. [29] showed that low VEGFR-1 tumor expression was associated with lower survival and correlated with an advanced disease status in breast cancer. According to these results, we expected CTCs ${ }^{\text {CK+VEGFR - }}$ patients to have a worse prognosis; however, we did not observe any difference in OS or PFS between patients with $\mathrm{CTCs}^{\mathrm{CK}+\mathrm{VEGFR}+}$ and CTCs${ }^{\mathrm{CK}+\mathrm{VEGFR}-}$. In this context, Senger et al. [30] demonstrated the existence of autocrine VEGF signaling in human tumors that might reflect the importance of VEGF for sustaining the self-sufficiency or autonomy of tumor cells, especially relevant to aggressive cancers and to the biology of cancer stem cells. Autocrine VEGF signaling is also closely associated with tumor dedifferentiation and with epithelial-mesenchymal transition (EMT), which are processes involved in the genesis of cancer stem cells. In this context, it has been suggested that autocrine signaling blocking by bevacizumab is modest and that its action can be improved by combining autocrine signaling blocking therapies [31]. These mentioned analyses, could explain why we cannot find differences in the OS and PFS between positive and negative VEGFR expression in CTCs.

The principal objective of this study was to compare CyCAR with RECIST to predict bevacizumab response. In our analysis, we observed no differences between both criteria. In fact, we detected similar results when we compared the CyCAR and the RECIST response at 12 weeks. We also obtained similar results when analyzing the assessment response at 12 (71\% vs. 93\%) and 24 weeks (82\% vs. $80 \%$ ) for CyCAR and RECIST respectively.

Our results suggest that the presence of CTCs at 12 and 24 weeks can be useful predictive markers, and used as a complementary tool with RECIST, even in those patients where RECIST can be more difficult to apply.

We also analyzed the predictive role of the presence of $\mathrm{CTC}^{\mathrm{CK}+\mathrm{VEGFR}+}$ to assess the treatment response in these patients. However, due to the low number of CTC CK+VEGFR+ patients we have not been able to demonstrate that the presence of VEGFR in CTCs is a predictive factor of tumor response to FOLFOX-bevacizumab. However, it would be important to note that one the principal limitation of this study is the methodology used to isolate these CTCs, since this isolation is based on the epithelial markers expression, therefore, we could be losing an important subpopulation of CTCs, which are under EMT process. Recently, Zhang et al. [32], demonstrated 
that VEGF expression rate in mesenchymal CTCs was significantly higher than that of epithelial CTCs, which suggested that VEGF may be correlated with tumor malignancy "and probably with the resistance".

Although these results suggest the value and importance of CTCs for monitoring treatments, further studies are necessary including, not only more patients, but also a deeper study about the autocrine and paracrine activity involved in the activation of VEGF and its role in the migration and proliferation of circulating tumor cells.

\section{Conclusion}

These results suggest that CyCAR is similar to RECIST criteria at evaluating the response in metastatic colorectal carcinoma. Although RECIST is useful for evaluating treatment efficacy in clinical trials and practice, it has some limitations. This way, we propose the use of CyCAR and RECIST combination for a better response prediction of metastatic colorectal carcinoma, in special when RECIST is limited.

\begin{abstract}
Abbreviations
Cl: confidence interval; CRC: colorectal cancer; CTC1: baseline CTCS; CTC2: CTCs at 12 weeks after treatment initiation; CTC3: CTCs at 24 weeks after treatment initiation; CTCs: circulating tumor cells; CyCAR: Cytologic Criteria Assessing Response; HR: hazard ratio; IF: immunofluorescence; mCRC: metastatic colorectal cancer; OS: overall survival; PFS: progression-free survival; RECIST: Response Evaluation Criteria in Solid Tumors; SD: standard deviation; VEGF-A: vascular endothelial growth factor $\mathrm{A}$.
\end{abstract}

\section{Authors' contributions}

Conceiving and designing the study: MJS, JAL, JLP. Processing and analyzing the biological samples: DdMP, FGO, AR. Developing the statistical analysis: MD. Analyzing and interpreting the data: MJS, MD, JEH. Writing the manuscript: MJS, JAL, MD, DdMP. Providing critical revisions: HI, MCGN. Approving the final version of the manuscript: HI, MJS. All authors read and approved the final manuscript.

\section{Author details}

${ }^{1}$ Integral Oncology Division, Clinical University Hospital, Av. Dr. Olóriz 16, 18012 Granada, Spain. ${ }^{2}$ Liquid Biopsy and Metastasis Research Group, GENYO, Centre for Genomics and Oncological Research, Pfizer/University of Granada/ Andalusian Regional Government PTS, Granada, Avenida de la llustración, 114 , 18016 Granada, Spain. ${ }^{3}$ Laboratory of Genetic Identification, Legal Medicine and Toxicology Department, Faculty of Medicine, University of Granada, Avenida de la Investigación, 11, 18071 Granada, Spain. ${ }^{4}$ DestiNA Genomics Ltd, 7-11 Melville St, Edinburgh EH3 7PE, UK. ${ }^{5}$ Division of Preventive Medicine and Public Health, CIBERESP, University of Jaen, Campus de las Lagunillas, 23072 Jaén, Spain.

\section{Acknowledgements}

We would like to extend our gratitude to the all the patients and the healthy volunteers who participated in the study.

\section{Competing interests}

The authors declare that they have no competing interests.

\section{Availability of data and materials}

Not applicable.

\section{Consent for publication}

Not applicable.
Ethics approval and consent to participate

This study was approved by the Independent Ethics Committee of San Cecilio Hospital and written consents were provided and signed by all patients.

\section{Funding}

This work was supported by Roche Spain and a Ph.D. grant from the University of Granada.

\section{Publisher's Note}

Springer Nature remains neutral with regard to jurisdictional claims in published maps and institutional affiliations.

Received: 25 June 2018 Accepted: 29 August 2018

Published online: 06 September 2018

\section{References}

1. Ferlay J, Soerjomataram I, Ervik M, Dikshit R, Eser S, Mathers C, et al. GLOBOCAN 2012 v1.0, Cancer Incidence and Mortality Worldwide: IARC CancerBase No. 11. Lyon: International Agency for Research on Cancer; 2014. http://globocan.iarc.fr. Accessed 19 June 2017

2. Edwards MS, Chadda SD, Zhao Z, Barber BL, Sykes DP. A systematic review of treatment guidelines for metastatic colorectal cancer. Colorectal Dis. 2012;14:e31-47.

3. Allen JE, El-Deiry WS. Circulating tumor cells and colorectal cancer. Curr Colorectal Cancer Rep. 2010;6:212-20.

4. Nadal R, Lorente JA, Rosell R, Serrano MJ. Relevance of molecular characterization of circulating tumor cells in breast cancer in the era of targeted therapies. Expert Rev Mol Diagn. 2013;13:295-307.

5. Lu CY, Tsai HL, Uen YH, Hu HM, Chen CW, Cheng TL, et al. Circulating tumor cells as a surrogate marker for determining clinical outcome to mFOLFOX chemotherapy in patients with stage III colon cancer. Br J Cancer. 2013;108:791-7.

6. Tanimoto K, Yoshiga K, Eguchi H, Kaneyasu H, Ukon K, Kumazaki T, et al. Hypoxia-inducible factor-1 a polymorphisms associated with enhanced transactivation capacity, implying clinical significance. Carcinogenesis. 2003;24:1779-83.

7. Mancuso MR, Davis R, Norberg SM, O'Brien S, Sennino B, Nakahara T, et al. Rapid vascular regrowth in tumors after reversal of VEGF inhibition. J Clin Invest. 2006;116:2610-21.

8. Kowanetz M, Ferrara N. Vascular endotelial growth factor signaling pathways: therapeutic perspective. Clin Cancer Res. 2006;12:5018-22.

9. Goel HL, Mercurio AM. VEGF targets the tumour cell. Nat Rev Cancer. 2013;13:871-82.

10. Hurwitz HI, Tebbutt NC, Kabbinavar F. Efficacy and safety of bevacizumab in metastatic colorectal cancer: pooled analysis from seven randomized controlled trials. Oncologist. 2013;18:1004-12.

11. Dy GK, Hobday TJ, Nelson G, Windschitl HE, O'Connell MJ, Alberts SR, et al. Long-term survivors of metastatic colorectal cancer treated with systemic chemotherapy alone: a North Central Cancer Treatment Group review of 3811 patients, n0144. Clin Colorectal Cancer. 2009;8:88-93.

12. Maru D, Venook AP, Ellis LM. Predictive biomarkers for bevacizumab: are we there yet? Clin Cancer Res. 2013;19:2824-7.

13. Eisenhauer EA, Therasse P, Bogaerts J, Schwartz LH, Sargent D, Ford R, et al. New response evaluation criteria in solid tumours: revised RECIST guideline (version 1.1). Eur J Cancer. 2009;45:228-47.

14. Serrano MJ, Ortega FG, Alvarez-Cubero MJ, Nadal R, Sanchez-Rovira P, Salido M, et al. EMT and EGFR in CTCs cytokeratin negative non-metastatic breast cancer. Oncotarget. 2014;5:7486-97.

15. Maldonado G, Greenland S. Simulation study of confounder-selection strategies. Am J Epidemiol. 1993;138:923-36.

16. Yang D, Woodard G, Zhou C, Wang X, Liu Z, Ye Z, et al. Significance of different response evaluation criteria in predicting progression-free survival of lung cancer with certain imaging characteristics. Thorac Cancer. 2016;7:535-42.

17. Nishino M, Jagannathan JP, Krajewski KM, O'Regan K, Hatabu H, Shapiro $\mathrm{G}$, et al. Personalized tumor response assessment in the era of molecular medicine: cancer-specific and therapy-specific response criteria to complement pitfalls of RECIST. AJR Am J Roentgenol. 2012;198:737-45. 
18. Cristofanilli M, Budd GT, Ellis MJ, Stopeck A, Matera J, Miller MC, et al. Circulating tumor cells, disease progression, and survival in metastatic breast cancer. N Engl J Med. 2004;351:781-91.

19. Ashworth TR. A case of cancer in which cells similar to those in the tumors were seen in the blood after death. Aust Med J. 1869;14:146-9.

20. Nadal R, Fernandez A, Sanchez-Rovira P, Salido M, Rodríguez M, GarcíaPuche $J L$, et al. Biomarkers characterization of circulating tumour cells in breast cancer patients. Breast Cancer Res. 2012;14:R71.

21. Dattatreya S. Metastatic colorectal cancer-prolonging overall survival with targeted therapies. South Asian J Cancer. 2013;2:179-85.

22. Das A, Kunkel M, Joudeh J. Clinico-pathological correlation of serial measurement of circulating tumor cells in 24 metastatic colorectal cancer patients receiving chemotherapy reveals interpatient heterogeneity correlated with CEA levels but independent of KRAS and BRAF mutation. Cancer Biol Ther. 2015;16:709-13.

23. Buim ME, Fanelli MF, Souza VS, Romero J, Abdallah EA, Mello CA, et al. Detection of KRAS mutations in circulating tumor cells from patients with metastatic colorectal cancer. Cancer Biol Ther. 2015;16:1289-95.

24. Tsai WS, Chen JS, Shao HJ, Wu JC, Lai JM, Lu SH, et al. Circulating tumor cell count correlates with colorectal neoplasm progression and is a prognostic marker for distant metastasis in non-metastatic patients. Sci Rep. 2016;6:24517.

25. Xu H, Xia Z, Jia X, Chen K, Li D, Dai Y, et al. Primary tumor resection is associated with improved survival in stage IV colorectal cancer: an instrumental variable analysis. Sci Rep. 2015;5:16516.
26. Tseng JY, Yang CY, Liang SC, Liu RS, Jiang JK, Lin CH. Dynamic changes in numbers and properties of circulating tumor cells and their potential applications. Cancers (Basel). 2014;6:2369-86.

27. Simiantonaki N, Taxeidis M, Jayasinghe C, Kirkpatrick CJ. Epithelial expression of VEGF receptors in colorectal carcinomas and their relationship to metastatic status. Anticancer Res. 2007;27:3245-50.

28. Hanrahan V, Currie MJ, Gunningham SP, Morrin HR, Scott PA, Robinson BA, et al. The angiogenic switch for vascular endothelial growth factor (VEGF)-A, VEGF-B, VEGF-C, and VEGF-D in the adenomacarcinoma sequence during colorectal cancer progression. J Pathol. 2003;200:183-94.

29. Lebok P, Huber J, Burandt EC, Lebeau A, Marx AH, Terracciano L, et al. Loss of membranous VEGFR1 expression is associated with an adverse phenotype and shortened survival in breast cancer. Mol Med Rep. 2016;14:1443-50

30. Senger DR, Van De Water L. VEGF expression by epithelial and stromal cell compartments: resolving a controversy. Am J Pathol. 2000;157:1-3.

31. Sullivan LA, Brekken RA. The VEGF family in cancer and antibody-based strategies for their inhibition. MAbs. 2010;2:165-75.

32. Zhang R, Xu J, Zhao J, Bai J. Expression of vascular endothelial growth factor in circulating tumor cells for prediction of colorectal cancer. Int J Clin Exp Pathol. 2017;10:5859-67.
Ready to submit your research? Choose BMC and benefit from:

- fast, convenient online submission

- thorough peer review by experienced researchers in your field

- rapid publication on acceptance

- support for research data, including large and complex data types

- gold Open Access which fosters wider collaboration and increased citations

- maximum visibility for your research: over 100M website views per year

At BMC, research is always in progress.

Learn more biomedcentral.com/submissions 